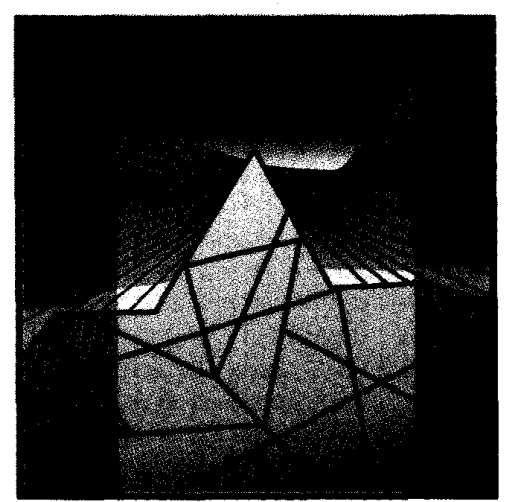

Valtino X. Afonso and Willis J. Tompkins Department of Electrical and Computer Engineering University of Wisconsin

\title{
Detecting Ventricular Fibrillation
}

\section{Selecting the appropriate time-frequency analysis fool for the application}

utomatic external defibrillators A(AEDs) require arrhythmia classification algorithms that can distinguish shockable cardiac rhythms from nonshockable cardiac rhythms. During ventricular fibrillation, the chaotic electrical activity in the myocardium results in diminished cardiac output, which if not treated can be fatal. AEDs should be able to detect shockable arrhythmias, distinguish these from nonshockable arrhythmias, and defibrillate the heart only when a shockable arrhythmia is detected.

Many algorithms to detect shockable rhythms have been reported in the literature [1]. Some algorithms use information from the time domain and others use information from the frequency domain. The performance of these algorithms is not ideal and each can be improved. The algorithms based on the frequency domain incorporate the short time Fourier transform (STFT) to compute the energy distribution of the electrocardiogram (ECG). Features are extracted from the energy distribution and are used in diagnostic classification algorithms [2]. The STFT is well known to have a tradeoff in resolution between time and frequency, and the features are thus limited by the accuracy of the frequency distribution. Accurate methods of computing the time-frequency distribution (TFD) need to be determined for arrhythmia classification algorithms. The objective of this article is to compare the TFD of normal and ventricular rhythms using such algorithms as the STFT, and time-frequency distributions such as the smoothed pseudo Wigner-Ville distribution (SPWVD), and the cone-shaped kemel (CKD) method. This is a pilot study to show that more discriminatory features can be extracted from the CKD or SPWVD than from the STFT for use in algorithms to distinguish VF and NSR.

\section{Shockable versus nonshockable rhythms}

The Association for the Advancement of Medical Instrumentation (AAMI), recommends that AEDs be able to distinguish

IEEE ENGINEERING IN MEDICINE AND BIOLOGY between shockable and nonshockable rhythms [3]. Shockable rhythms include ventricular fibrillation (VFib) and possibly ventricular tachycardia (VT). Nonshockable rhythms include all other arrhythmias including normal sinus rhythm (NSR), atrial fibrillation, idioventricular rhythms, and asystole. Figures 1 and 2 show examples of NSR and VFib respectively. Figure 3 shows an ECG that changes rhythm from VT to VFib.

Time-frequency distributions

There are a number of methods for transforming a 1-D signal in time into a 2-D distribution of signal strength in time and frequency [4]. The TFD gives a measure of the intensity of frequencies over time. Various transformation methods have certain properties that may have benefit in different applications $[4,5]$. Some transformation methods such as the Wigner distribution provide very good resolution in time and frequency of the underlying structure of the signal. However, in the case of signals with more than one frequency at any time instant (e.g., the ECG), an inaccurate TFD results due to the presence of cross terms or interference terms [6]. These are spurious frequencies which show up in the TFD and are not due to the real signal.

The TFD obtained using the STFT method has a tradeoff between time and frequency resolution. Increased resolution in frequency requires a longer data segment of the ECG. However, in the case of ECG signals, longer data segments have a frequency distribution that varies in time. Increased time resolution thus requires a shorter window length. Other methods of computing the TFD include the CKD method and the SPWVD, which are designed to reduce the cross terms in the TFD.

None of these methods work well for all types of signals, and furthermore, none were designed explicitly for analyzing the ECG. However, a comparison of various methods of time-frequency analysis will indicate better methods for the ECG than 


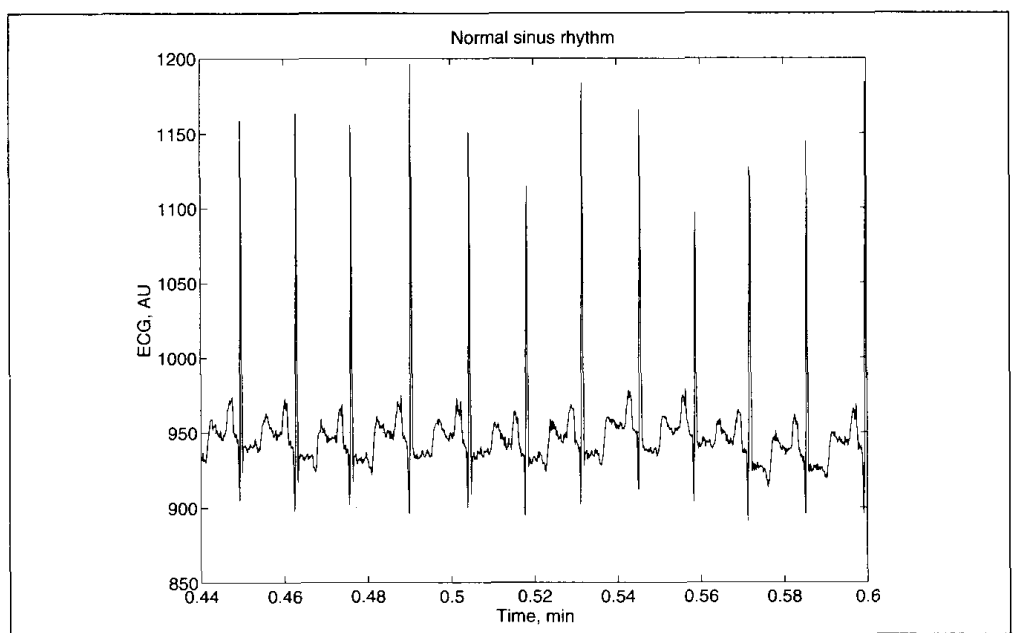

1. An example of normal sinus rhythm.

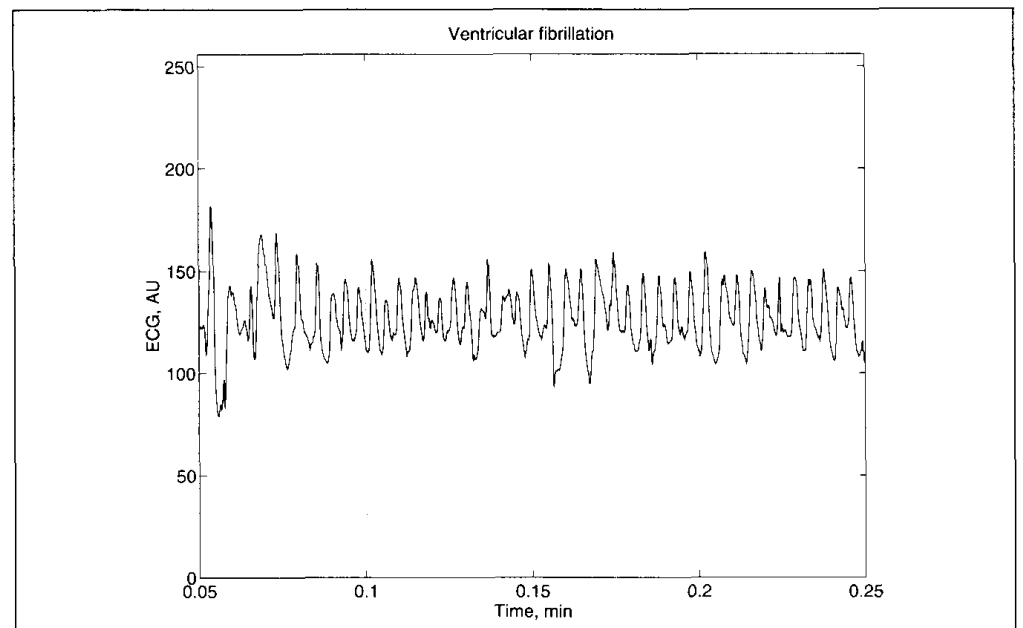

2. An example of ventricular fibrillation.

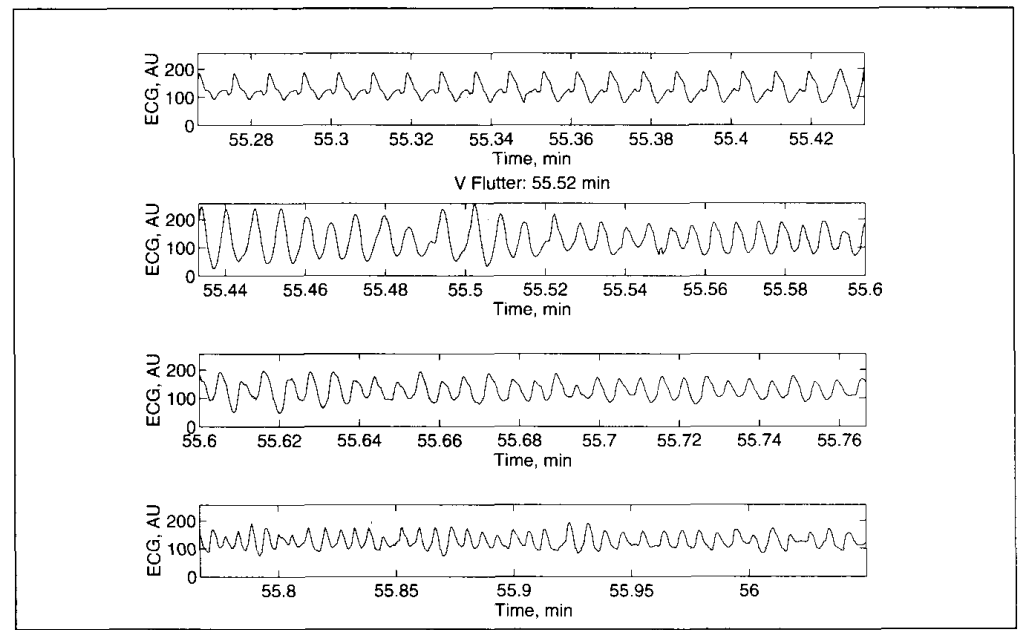

3. The electrocardiogram changes rhythm from ventricular tachycardia to ventricular flutter to ventricular fibrillation. the more commonly used STFT, and also the need for improved methods of computing TFDs.

\section{Discriminatory feature}

Arrhythmia classification algorithms incorporate various features from the time and frequency domain of the ECG [1]. The frequency domain of a normal sinus rhythm signal has harmonics that cover a wide range of frequencies. The frequency domain of VFib signals covers a narrower range of frequencies of about $4-7 \mathrm{~Hz}[1$, 2]. It is thus useful to extract features from the frequency domain to classify arrhythmias. A VFib detection algorithm reported in [2] uses the fast Fourier transform to compute the energy of the signal from a segment of the ECG. First, the frequency

$F_{m}$ with the maximum energy is determined. Then, the normalized energy in the frequency band from $0.7 F_{m}$ to $1.4 F_{m}(\mathrm{~Hz})$ is computed. Finally, the normalized energy in the harmonics of $F_{m}$ is computed These features are chosen to determine the predominant frequency in the signal and the relative energy in this frequency band, and serve as a measure of the characteristics of VFib and normal rhythms in the frequency domain.

In order to extract features from the frequency domain, it is important to have an accurate frequency representation of the signal. Since it is also important to detect VFib and other arrhythmias as soon as they occur, optimal time resolution is necessary. An optimal method of computing the time-frequency distribution for use in arrhythmia classification needs to be determined.

\section{Time-Frequency Distributions}

Time-frequency analysis such as the short time Fourier transform, Wigner distribution, smoothed pseudo Wigner-Ville distribution, and cone-shaped kernel are described in the literature $[4,6,7,9]$. The properties of each these time-frequency analysis methods are already described in great detail elsewhere. The main goal of this article is to compare these different methods for potential use in arrhythmia classification algorithms. For the sake of completeness, a short explanation is given of each method and its relevant parameters.

\section{Short time Fourier transform} (STFT)

Let $g(t)$ be a finite length window, and $g(t '-t)$ the same window centered about time $t$. The STFT is defined as:

$$
\operatorname{STFT}(t, f)=\int x\left(t^{\prime}\right) \gamma^{*} e^{-j 2 \pi t^{\prime}} d t^{\prime}
$$

Note that the STFT computes the strength 
of frequencies in the signal around time $t$. The spectrogram can then be obtained by squaring the STFT modulus. Properties of this transformation are discussed in $[4,5$, 7].

The window, $\gamma(t)$, suppresses the signa outside a neighborhood around the analysis time point $t^{\prime}=t$, and thus the STFT gives a localized measure of the strength of the signal's frequencies around the time $t$. The filter bank interpretation [4] helps in visualizing the STFT as a bank of bandpass filters, each centered around the frequency being evaluated. The signal $x(t)$ passes through each bandpass filter, which is essentially the FT of the window $\gamma(t)$. Optimal frequency resolution requires that the bandwidth of the bandpass filter be arbitrarily small. This is accomplished if the window $\gamma(t)$ has infinite length. However, in order to have good time resolution, the window $\gamma(t)$ should have a short length. The obvious limitation of the STFT is that it is not possible to have both optimal time resolution and optimal frequency resolution at the same time. Since the STFT depends only on one window, its time and frequency resolutions cannot both be high. This is the main disadvantage of the STFT. The other distributions described below attempt to improve on the time-frequency resolution of a signal by introducing a quadratic function with respect to the input signal.

\section{Wigner distribution (WD)}

The Wigner distribution has very high resolution in time and frequency $[4,7]$. It is defined as:

$$
\begin{aligned}
& W D_{x}(t, f)= \\
& \int x\left(t+\frac{\tau}{2}\right) x *\left(t-\frac{\tau}{2}\right) e^{-j 2 \pi f \tau} d \tau
\end{aligned}
$$

The WD is a quadratic distribution that satisfies many desirable properties of TFDs. However, for multicomponent signals (i.e., those with more than one frequency), there are interference terms, also called cross terms, which cause problems in the interpretation of the TFD. For example, if there is a signal at $t_{1}$ with frequency $f_{1}$ and another signal at $t_{2}$ with frequency $f_{2}$, then there is a cross term present at time $t_{12}=\left(t_{1}+t_{2}\right) / 2$, with frequency $f_{12}=\left(f_{1}+f_{2}\right) / 2$. The literature provides an analysis of how the cross terms arise [4].

Although the WD provides improved time and frequency resolution, the presence of the cross terms is a disadvantage. The cross terms show up as intensities in time and frequency that are not truly present in the signal. A feature such as the energy in a frequency band will be affected by the cross terms and not the signal itself. This feature will lead to an incorrect classification of the ECG. Thus, the main disadvantage of the WD is the presence of cross terms.

\section{Smoothed pseudo Wigner-Ville Distribution (SPWVD)}

A variant of the WD incorporates smoothing the TFD by independent windows in time and frequency [6]:

$S P W V D_{x}(t, f)=\int W_{f}(\tau) \times$

$\left[\int W_{t}(u-t) x\left(u+\frac{\tau}{2}\right) x *\left(u-\frac{\tau}{2}\right) d u\right] e^{-j 2 \pi f \tau} d \tau$

The two windows, $W_{t}$ and $W_{f}$, independently determine the amount of smoothing in time, and frequency, respectively. The choice of the length of the windows determines the amount of cross term suppression obtainable. The lengths of the windows must be determined empirically for the type of signals being analyzed $[4,6]$.

\section{Cone-shaped kernel distribution (CKD)}

The CKD offers a method of obtaining improved time and frequency resolution for nonstationary signals with reduced cross terms [9]. The CKD is given by:

$$
\begin{array}{r}
C K D(t, f)=\iint \varphi(t-u, \tau) x\left(u+\frac{\tau}{2}\right) \\
x^{*}\left(u-\frac{\tau}{2}\right) e^{-j 2 \pi \tau} d u d \tau
\end{array}
$$

The above equation is a generalized expression for computing a TFD. Different methods of computing the TFD design the kernel $\varphi()$, such that the TFD meets many desired properties. Some of these properties include nonnegativity, maintaining time and frequency marginals, finite support, and reduced interference terms [5,7]. The cone-shaped kernel is designed as a lateral inhibition function. Thus, when computing the intensity of the signal at a particular frequency, a small neighborhood around this frequency contributes positively, while frequencies outside the neighborhood contribute negatively. The cone-shaped kernel is given by

$$
\begin{array}{cl}
\varphi(t, \tau)=g(\tau) & |\tau| \geq a|t| \\
0 & \text { otherwise }
\end{array}
$$

where $2 \geq a<\infty$.

A discretized algorithm given in [9] is used in this study. The CKD does not satisfy the nonnegative property of TFDs, but it does have good cross term suppression.

\section{Methods}

\section{ECG database}

This study uses the Staley cardiac arrhythmia database [10]. The raw data for this database was acquired by the Wisconsin-Dane County EMT-defibrillation program, and includes multiple recordings of ventricular fibrillation, asystole, and other rhythms. Lead II of the ECG was acquired with a semiautomatic defibrillator (Marquette, Inc., Milwaukee, WI, model Responder 1200) at a sampling rate of 100 $\mathrm{Hz}$ and 8-bit amplitude resolution. A SRAM card in the defibrillator stored the sampled ECG until it was downloaded to a PC. Physicians, comments, and interpretations of the ECG recordings were then added to the database. Since the Staley database consists of actual abnormal ECG recordings collected on emergency runs, it is useful for the development and testing of ECG processing or monitoring algorithms and instruments.

\section{Signal preprocessing}

We extracted normal sinus rhythm (NSR) signals from the MIT/BIH database, which has a sampling rate of $360 \mathrm{~Hz}$, as opposed to $100 \mathrm{~Hz}$ for the Staley database. Then, the NSR signals were downsampled to $100 \mathrm{~Hz}$ using software provided with the MIT/BIH database. We extracted recordings of VFib, VFlut, and VTach signals from the Staley database.

After this, we processed all the ECG signals with a bandpass filter using $2 \mathrm{~Hz}$ and $20 \mathrm{~Hz}$ as cutoff frequencies. The filter design used a MATLAB ${ }^{\circledR}$ command, which implements the window method. Based on a Hamming window, the order of the filter was 61 . The window used is not important in this study as long as the filter has linear phase and the attenuation outside the frequency range of interest is reasonably large. The cutoff frequencies for the bandpass filter were chosen based on studies of the frequency domain of ECG signals, in particular VFib signals [11], and features of the frequency domain used in VFib detection algorithms [2].

\section{Computing TFDs}

The parameters in the different methods of computing the TFD were determined by first testing the methods on an artificial signal with frequencies similar to that of a VFib signal. An artificial signal was generated with a $100 \mathrm{~Hz}$ sampling rate. The signal constituted a $5-\mathrm{Hz}$ sinusoid from $0-0.5 \mathrm{~min}$, a $6-\mathrm{Hz}$ sinusoid from $0.45-0.56 \mathrm{~min}$, and a $7-\mathrm{Hz}$ sinusoid from $0.52-1 \mathrm{~min}$. We chose the frequencies of the sinusoids to be in the expected 


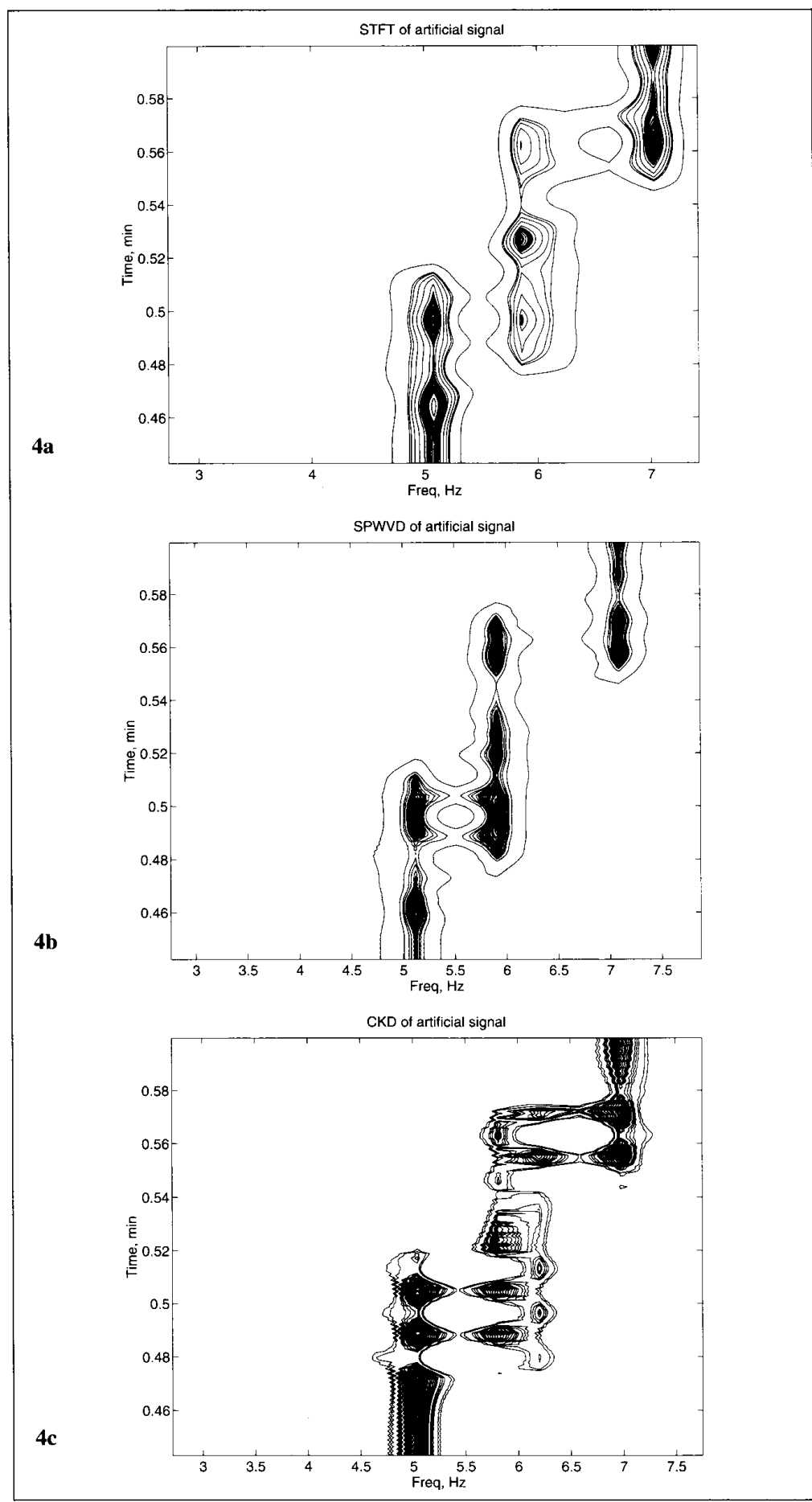

4. (a) Contour plot of the STFT is given for the artificial signal. The outermost contour is drawn at a level of half of the average of maximum intensities in the time-frequency distribution. The contours are not as closely spaced as in the SPWVD (b), and CKD (c). Contour plot of the SPWVD (b) is given for the artificial signal. The contours are more closely spaced than in the STFT (a). Contour plot of the CKD (c) is given for the artificial signal. The contours are more closely spaced than in the STFT (a). range of VFib signals [11]. We can determine frequency resolution because of the coexisting sinusoids. We can determine the ability to detect onset of the signals since the sinusoids are time limited.

Using a Hanning window based STFT, the contour plots showed best time and frequency resolution using $2.56 \mathrm{~s}(0.043$ min) data segments. In order to do a fair comparison of the STFT with the SPWVD and CKD methods, the length of the data segment for each method was kept as similar as possible. The SPWVD used a 2.59 $\mathrm{s}$ data segment with time and frequency smoothing windows each of $1.28 \mathrm{~s}$. The CKD method also used a $2.59 \mathrm{~s}$ data segment.

At each time instant, the previous 2.56 $\mathrm{s}$ (or $2.59 \mathrm{~s}$ for SPWVD and CKD) of data is transformed into the frequency domain, a fact that must be clear when analyzing the TFDs and accounting for the delay inherent in the time-frequency analysis. The time axis of the contour plots in this article indicates the time each transformation was computed. The STFT, SPWVD and CKD methods of computing the TFD were implemented using a Hewlett Packard RISC 700 series computer with the $\mathrm{C}$ and MATLAB programming languages.

\section{Contour plots}

The 1-D signal in time, the ECG, is transformed using different methods into a 3-D plot of signal intensity as a function of time and frequency. Based on the method of transformation, the 3-D TFD of the signal manifests to varying degrees of accuracy the true time-frequency structure of the ECG.

It is difficult to visualize these 3-D plots from one 2-D representation. Thus, we have presented certain features of the 3-D TFD by drawing contour plots. For a given TFD, the maximum intensity is noted over all frequencies for each time instant (or time slice). Similar maximum intensities are recorded for each time slice in the TFD. These maximum intensities are sorted in descending order of magnitude and the average is also computed

Using the sorted values of maximum intensities, contours are drawn on the 3-D TFD at levels ranging from the maximum intensity down to the lowest intensity, skipping every few values along the way. A contour is also drawn at the level of half of the average of the maximum intensities. This contour gives an indication of the dynamic range of the intensity of the signal in time and frequency. A narrow contour indicates good time-frequency resolution, as compared to a wide contour. 


\section{Results}

\section{TFD contour plots}

of artificial signal

The contour plots of the TFD for the artificial signal are given in Fig. 4. The time axis denotes the time at which the transformation to the frequency domain was computed by using data collected up to that time. Thus in Fig. 4a, the time slice at $0.46 \mathrm{~min}$ was computed from the previous $2.56 \mathrm{~s}$ of a $5 \mathrm{~Hz}$ signal, with only 0.6 $\mathrm{s}(0.1 \mathrm{~min})$ of the $6 \mathrm{~Hz}$ signal. Recall that the artificial signal was made up of a $5 \mathrm{~Hz}$ signal from $0-0.5 \mathrm{~min}$, a $6 \mathrm{~Hz}$ signal from $0.45-0.56 \mathrm{~min}$, and a $7 \mathrm{~Hz}$ signal from $0.52-1.0 \mathrm{~min}$.

The contour plot from the STFT algorithm shown in Fig. 4a has less resolution in the frequency domain than that of the SPWVD in Fig. 4b, and of the CKD algorithms in Fig. 4c. For example, the time slice at 0.46 min shows a wider spread of the contours in frequency than do the SPWVD and CKD algorithms. The time slice at about 0.5 min indicates that the SPWVD algorithm can resolve the $5 \mathrm{~Hz}$ and $6 \mathrm{~Hz}$ sinusoids better than the STFT. Note that the proximity of the contours indicates the intensity of each sinusoid in the TFD contour plot.

There are spurious cross terms that show up in the CK contour plot and, to a lesser extent, in the SPWVD contour plot. For example, in Figs. $4 b$ and $4 c$, the time slice at about $0.505 \mathrm{~min}$ shows cross terms between the true frequencies of 5 and 6 Hz. The STFT does not manifest these cross terms. However, even at this time instant, the resolution of the true sinusoids is still lowest in the STFT shown in Fig. $4 \mathrm{a}$.

Time resolution can be compared by studying the plots in Fig. 4 around the time of $0.55 \mathrm{~min}$ and frequency of $7 \mathrm{~Hz}$. At this time slice, the STFT of Fig. 4a has less densely-spaced contours as in the SPWVD of Fig. 4b, and the CKD of Fig. $4 \mathrm{c}$. This indicates that the $7 \mathrm{~Hz}$ sinusoid is detected sooner in the SPWVD and CKD than in the STFT. As noted above, there is an inherent delay in all the algorithms. Even though the $7 \mathrm{~Hz}$ signal begins at 0.52 min, the time slice of the TFD at $0.55 \mathrm{~min}$ was computed using only about $0.03 \mathrm{~min}$ (0.55 - $0.52 \mathrm{~min}$ ), of the $7 \mathrm{~Hz}$ sinusoid.

\section{TFD contour plots of} normal sinus rhythm

Figure 1 shows an ECG with a normal sinus rhythm. The contour plots of the STFT, SPWVD, and CKD are shown in Figs. 5a, 5b, and 5c, respectively. As stated above, the time axis indicates the time of the end of the data segment used in the transformation to the frequency domain.

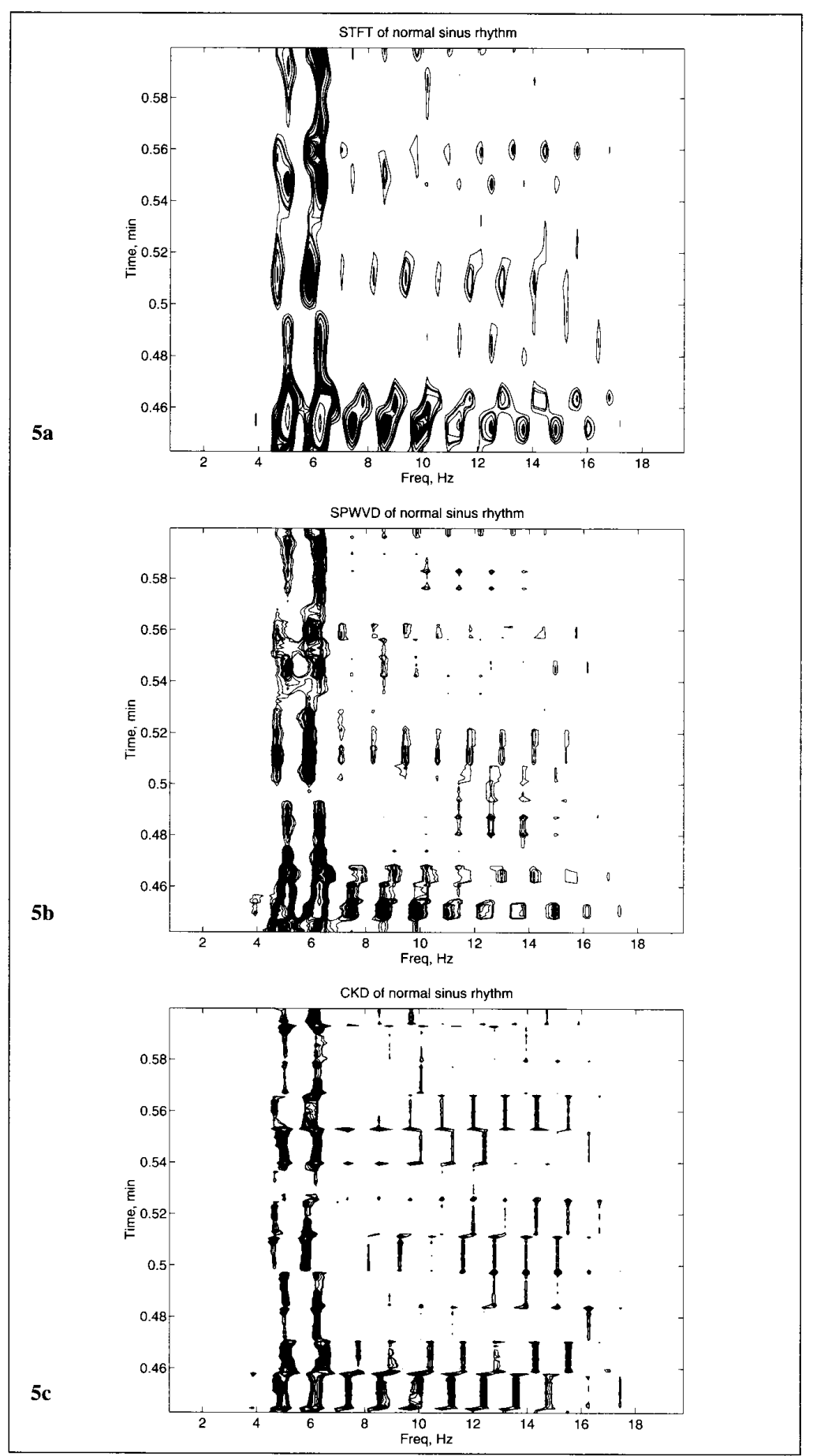

5. (a) Contour plot of the STFT for normal sinus rhythm. Harmonics characteristic of NSR are observed. This plot should be compared with the SPWVD (b) and CKD (c). Contour plot of the SPWVD (b) for normal sinus rhythm. This plot shows higher time-frequency resolution than the STFT (a). However, cross terms are also visible around the time of $0.54 \mathrm{~min}$ and frequency of $5 \mathrm{~Hz}$. Contour plot of the CKD (c) for normal sinus rhythm. This plot shows higher time-frequency resolution than the STFT (a). Features such as the energy in the harmonics of the peak frequency will be more discriminatory than from the STFT. 


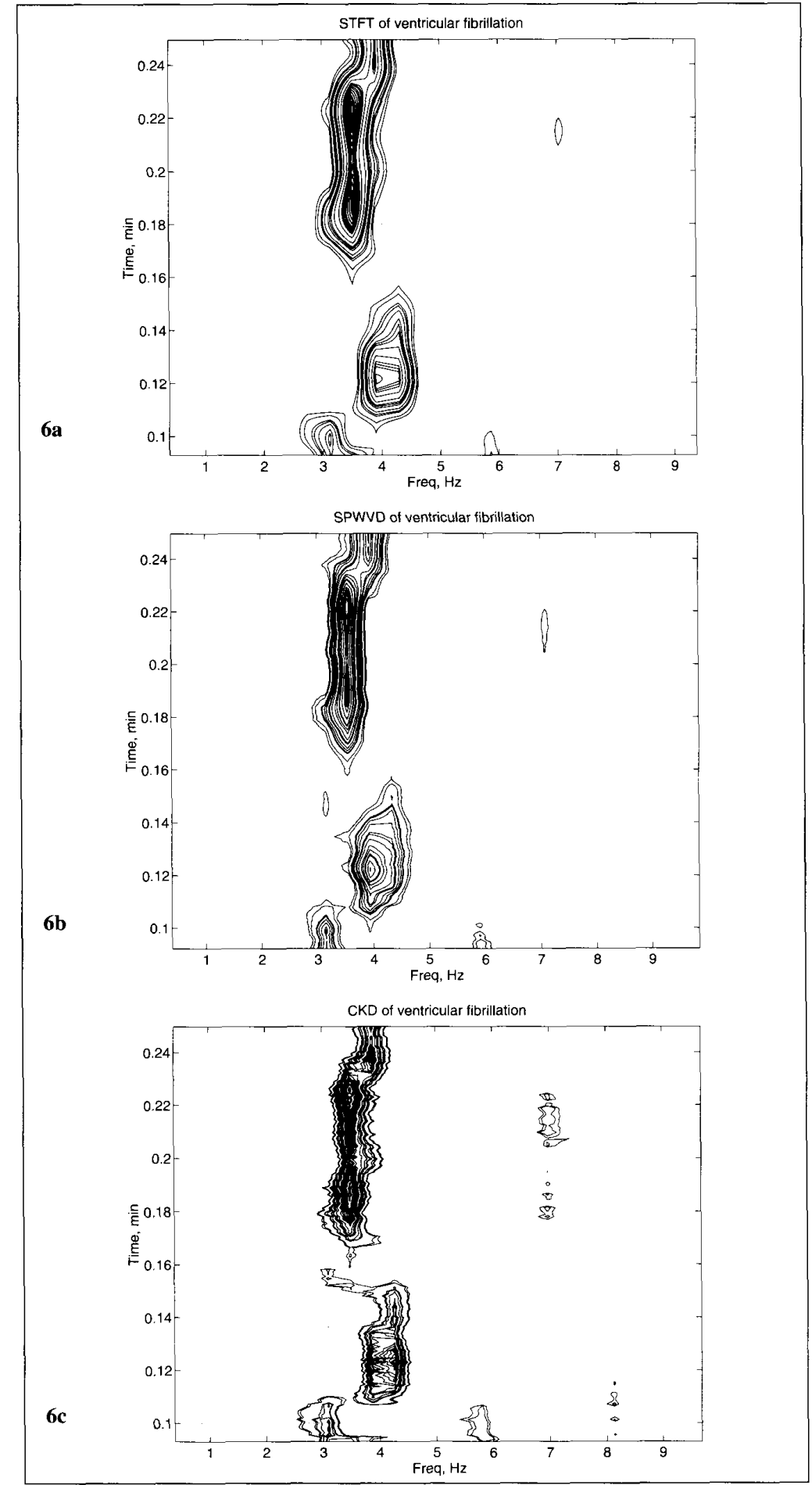

6. (a) Contour plot of the STFT for ventricular fibrillation. The characteristic one dominant frequency is observed. Contour plot of the SPWVD (b) for ventricular fibrillation. The contours are more closely spaced than in the STFT (a), indicating better time-frequency resolution. Contour plot of the CKD (c) for ventricular fibrillation. The contours are more closely spaced than in the STFT (a) and SPWVD (b), indicating better time-frequency resolution.
All the TFDs show the harmonics in the frequency domain that are characteristic of NSR. However, the proximity of the various contour levels is different for the different TFDs. The frequency resolution of the STFT is not as high the SPWVD and the CKD. For example, the time slice at about 0.465 min has contours that are most closely spaced in the CKD and comparable in the SPWVD. The proximity of the contours in the TFD indicates that there is a peak in the intensity of the signal for that time and frequency. However, the STFT at the time slice $0.465 \mathrm{~min}$ does not show such a high resolution in frequency.

Similarly, around $0.465 \mathrm{~min}$ and a frequency of $14 \mathrm{~Hz}$, the resolution in time can be compared. Contours from the SPWVD and CKD show more closely spaced contours than those of the STFT. At about this frequency, $14 \mathrm{~Hz}$, the signal intensity around time $0.465 \mathrm{~min}$ is more prominent in the CKD plot than in the STFT plot. The contour plot of the CKD shows the harmonics of NSR signal more remarkably than the SPWVD and STFT.

TFD contour plots of ventricular fibrillation

Figure 2 shows an example of a ventricular fibrillation rhythm. The contour plots of the STFT, SPWVD, and CKD are given in Figs. 6a, 6b, and 6c, respectively. The time axis indicates the end of the data segment used for computation of each time slice in the TFD, and thus the TFD can be compared with the real VFib signal shown in Fig. 2

The contour plots of all the TFDs show similar time-frequency structures for the VFib signal. However, the contour plots of the SPWVD and CKD show an increased time and frequency resolution compared to the STFT. This is manifested by the proximity of the contours in each of the plots. For example, within the $3-4 \mathrm{~Hz}$ frequency range, and during the $0.20-0.22$ min time interval, the contours cover a narrower range in frequency in the SPWVD and the CKD than in the STFT.

TFD contour plots during rhythm changes

Figure 3 shows a ventricular rhythm that changes from tachycardia to flutter to fibrillation. The contour plots for the STFT, SPWVD, and CKD are given in Figs. $7 \mathrm{a}, 7 \mathrm{~b}$, and $7 \mathrm{c}$, respectively.

During the VTach rhythm, two frequencies are present. When the rhythm progresses to VFlut (around $55.52 \mathrm{~min}$ ), there is a range of frequencies that is manifested with more frequency resolution in the contour plot of the CKD than that of the STFT. The SPWVD, on the other hand, shows some spurious frequencies, 
which may be a result of the cross terms. During VFib, the characteristic one-frequency pattern is manifested again. However, the variation in this one-frequency pattern is more discernible in the CKD than in the STFT.

\section{Discussion}

The contour plots of the STFT, SPWVD, and CKD of the ECG signals shown above manifest a similar time-frequency structure. However, as stated above, further processing is usually performed on the TFDs to extract features that may characterize the ECG rhythm. Examples of these parameters are the frequency of maximal intensity, $F_{\mathrm{m}}$ (peak frequency), the normalized energy in the peak frequency band defined around $F_{\mathrm{m}}$, and the normalized energy in the harmonics of $F_{\mathrm{m}}$. It is crucial that these features be extracted from an accurate time-frequency structure of the signal.

For example, during VFib the normalized energy in the peak frequency band would be different if obtained from the STFT (Fig. 6a) than if obtained from the CKD (Fig. 6c). Similarly, during ventricular flutter (Fig. 3, about $5.52 \mathrm{~min}$ ), the CKD (Fig. 7c) shows a different pattern than the STFT (Fig. 7a). Features such as the peak frequency, normalized energy in the peak frequency band, and normalized energy in the harmonics of the peak frequency would be more discriminatory using the CKD than the STFT.

By comparing the contour plots from the various arrhythmias, we note that tracking high intensity frequencies over time would be useful for an arrhythmia classifier. Most VFib detection algorithms reported in the literature incorporate frequency domain information from a particular time slice in the TFD. But it is apparent from the contour plots of the CKD that tracking frequencies in time will be useful to arrhythmia classification.

However, a very natural step, or an unfortunate reflex, of the many time-frequency algorithms available in the literature is to implement one or more of them, extract some or many features from the resulting TFDs, and then use them in classification algorithms. The various methods of time-frequency analysis reported in the literature are designed to meet desirable mathematical properties. Many of these mathematical properties such as the nonnegativity of a TFD are important. But it is equally important to design an optimal TFD for a specific application, which in this case, is arrhythmia analysis. Thus an accurate TFD should first be obtained, and then more discriminatory features can be extracted for classifying algorithms.

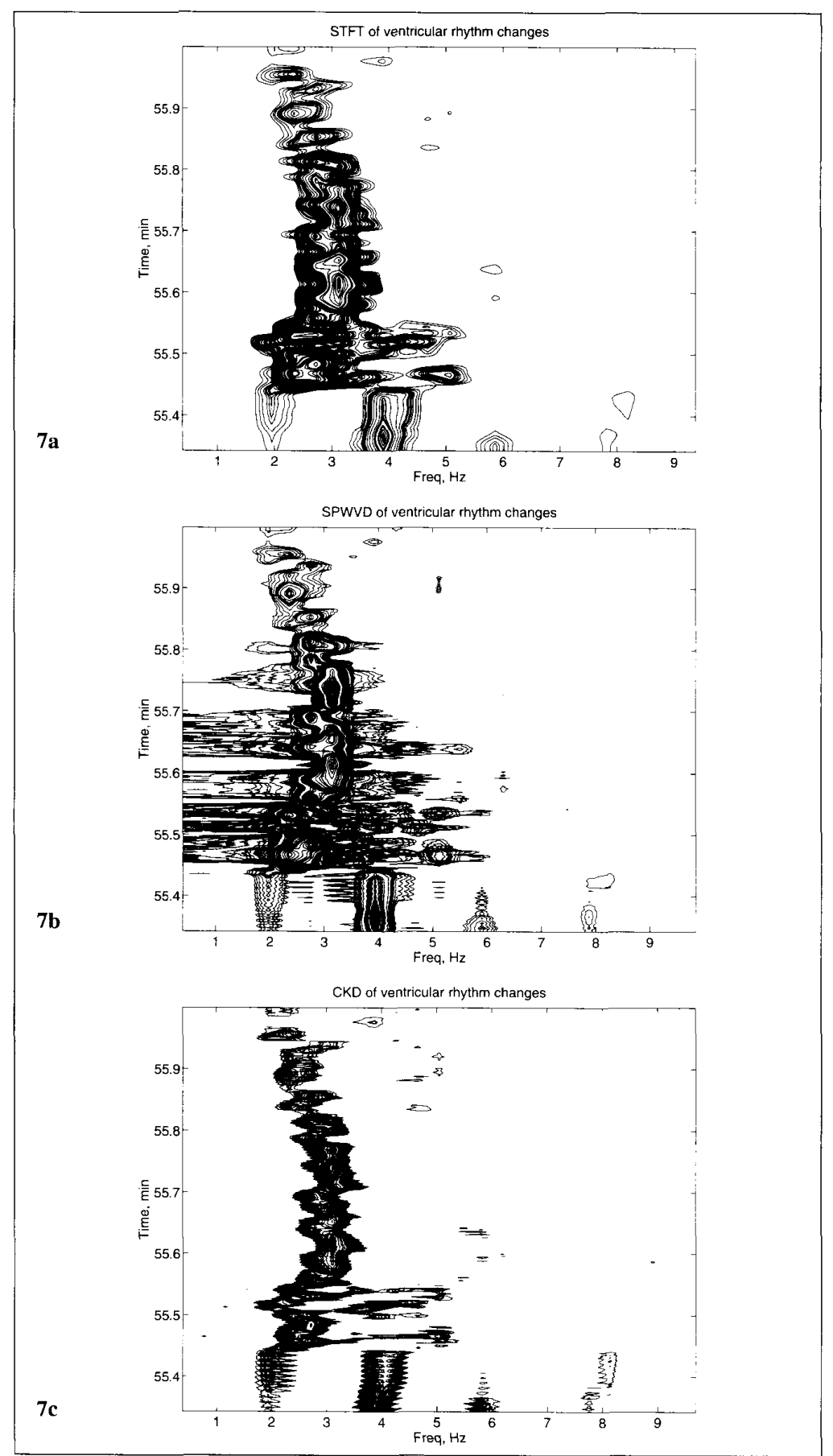

7. (a) Contour plot of the STFT for changes in ventricular rhythm from tachycardia to flutter to fibrillation. The time-frequency structure changes from two predominant frequencies characteristic of ventricular tachycardia to one predominant frequency characteristic of ventricular fibrillation. (b) Contour plot of the SPWVD for changes in ventricular rhythm from tachycardia to flutter to fibrillation. Spurious cross terms are observed in the TFD. (c) The contour plot of the CKD for changes in ventricular rhythm from tachycardia to flutter to fibrillation. The TFD shows features of the time-frequency structure with better resolution than the STFT (a) and SPWVD (b). 


\section{Summary}

In this article, the short time Fourier transform, smoothed pseudo Wigner Ville distribution, and cone-shaped kernel distribution have been used to compare the time-frequency distribution of normal sinus rhythm, ventricular tachycardia, ventricular flutter, and ventricular fibrillation signals. This work is a pilot study to illustrate that the CKD and SPWVD have better time and frequency resolution than the STFT. It demonstrates that accurate methods of computing the time-frequency domain should be found for ECG signals. Only then should future work be done to design discriminatory features and classifiers for arrhythmias.

\section{Acknowledgment}

This work was supported by Burdick Corp., Milton, WI. Recordings and interpretation of the arrhythmia database were provided by Dr. R. L. Staley, who is with the Dane County Emergency Service, Madison, WI. The authors also wish to thank the many useful viewpoints offered by Prof. Truong Nguyen.

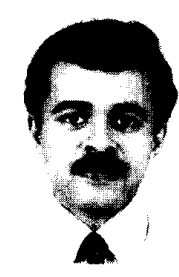

Valtino $X$. Afonso received the B.S. degree in electrical engineering from Marquette University, Milwaukee, WI in 1991, and the MSEE from the University of Wisconsin, Madison, in 1993. He is presently doing graduate work at the University of Wisconsin, and is pursuing the Ph.D de- gree. His research interests include timefrequency analysis of biomedical signals, and the application of signal processing to biomedical problems.

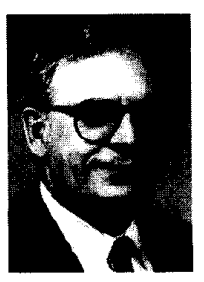

Willis J. Tompkins, an IEEE Fellow, received the B.S. and M.S degrees in electrical engineering from the University of Maine in 1963, and 1965, respectively, and the Ph.D degree in biomedica electronici engineering from the University of Pennsylvania, Philadelphia, in 1973. He has been on the faculty of the University of Wisconsin, Madison since 1974. Currently, he is Professor and Chair of the Department of Electrical and Computer Engineering. His research interests include applications of microcomputerbased medical instrumentation and online biomedical computing. Dr. Tompkins is a Past President of the IEEE Engineering in Medicine and Biology Society.

The authors may be reached at the Department of Electrical and Computer Engineering, University of Wisconsin, 1415 Johnson Drive, Madison, WI 53706.

\section{References}

1. Clayton RH, Murray A, Campbell RWF: Comparison of four techniques for recognition of ventricular fibrillation from the surface ECG. Med. and Biol. Eng. and Computing 31:111-117, 1993.

2. Barro S, Ruiz R, Cabello D, Mira J: Algo- rithmic sequential decision-making in the frequency domain for life threatening ventricular arrhythmias and imitative artefacts: a diagnostic system. J. Biomed. Eng. 11, 320-328, 1989.

3. Association for the Advancement of Medical Instrumentation: Automatic external defibrillators and remote-control defibrillators. ANSI/AAMI DF39, 1993.

4. Hlawatsch F, Boudreaux-Bartels GF: Linear and quadratic time-frequency signal representations. IEEE Sig. Proc. Mag. 21-67, April 1992.

5. Loughlin PJ, Pitton JW, Atlas LE: Bilinear time-frequency representations: new insights and properties. IEEE Trans. on Sig. Proc. 41(2):750$766,1993$.

6. Flandrin P: Some features of time-frequency representations of multicomponent signals. Proc IEEE Int. Conf. Acoust, Speech, Signal Processing 4IB:4.1-4.4, 1984.

7. Jeong J, Williams WJ: Kernel design for reduced interference distributions. IEEE Trans. on Sig. Proc. 40(2):402-412, 1992.

8. Jones DL, Parks TW: A resolution comparison of several time-frequency representations. IEEE Trans. on Sig. Proc. 40(2):413-420, 1992.

9. Zhao Y, Atlas LE, Marks RJ: The use of cone-shaped kernels for generalized time-frequency representations of nonstationary signals IEEE Trans. on Acoust., Speech, and Sig. Proc 38(7): 1084-1091, 1990

10. Panescu D, Webster JG, Tompkins WJ, Staley RL, Johnson J, et al: A database of cardiac arrhythmias. Academic Emergency Medicine. To appear November or December 1994.

11. Nolle FM, Bowser RW, Badura FK, Catlett JM, Gudapati RR, et al: Evaluation of a frequency-domain algorithm to detect ventricular fibrillation in the surface electrocardiogram. IEEE Computers in Cardiology. 337-340, 1994 